\title{
Dental Appliances and Mandibular Advancement Devices in Obstructive Sleep Apnea
}

\author{
Viranchi Oza
}

International Journal of Head and Neck Surgery (2019): 10.5005/jp-journals-10001-1366

\section{Case Description}

A 46-year-old overweight male (BMI 32) was diagnosed with moderate obstructive sleep apnea (OSA). He was recommended weight loss and asked to put on continuous positive airway pressure (CPAP) therapy. Follow-up visits failed to show a significant improvement in OSA. Sleep study indicated that he used CPAP for only 3 hours a night. He revealed that he does not like using CPAP. The sleep physician recommended a custom-made mandibularrepositioning oral appliance. Further follow-up visits revealed considerable improvements in his OSA symptoms, and visits to the dentist were scheduled to titrate the appliance as required.

An OSA has a high prevalence. The standard treatment for patients with OSA has been weight loss and use of nasal CPAP, although some cases require upper airway reconstructive surgery. However, adherence to CPAP therapy is very low, with the long-term adherence rate being as low as $50 \% .{ }^{1}$ Therefore, alternatives to CPAP and surgery are in great need. Oral appliances (OAs) constitute a good alternative for patients with mild to moderate OSA. ${ }^{2}$

The OAs are devices that are fitted in the mouth during sleep to prevent the oropharyngeal tissues and the base of the tongue from collapsing and obstructing the upper airway. Dr Pierre Robin was one of the first doctors to use dental appliances for glossoptosis. ${ }^{3}$ Later, adjustable mandibular advancement appliances were used for the management of sleep breathing disorder. Over the last few decades, there have been many advances in the design of the OAs, making them lighter and less cumbersome; these improvements have contributed to enhanced compliance of the patients. Since the inclusion of OA therapy in the guidelines of the American Academy of Sleep Medicine (AASM) in 1995, use of OAs has been steadily increasing. Recent advances in the treatment of OSA with dental appliances include sensors for monitoring the efficacy and fabrication of lighter appliances via 3D printing technology. In India, however, awareness among clinicians regarding OAs continues to below.

Meta-analyses revealed that OAs decrease apnea-hypopnea index (AHI) across all levels of OSA severity comparable to that achieved by CPAP. ${ }^{4}$ OAs can be used in patients with primary snoring (without OSA) (1) as well as in patients with mild to moderate OSA who prefer them to or have low compliance to CPAP therapy and in those who do not respond to or are not appropriate candidates for CPAP therapy. In addition, patients in whom behavioral measures such as weight loss, avoidance of alcohol and smoking, and sleep position change have partly or completely failed are also good candidates for OAs. ${ }^{5}$ Other indications of OA therapy include the following:
Dentistry and Dental Sleep Medicine, Viheet Dental and Sleep Clinic, India

Corresponding Author: Viranchi Oza, Dentistry and Dental Sleep Medicine, Viheet Dental and Sleep Clinic, India, e-mail: viranchioza@ gmail.com

How to cite this article: Oza V. Dental Appliances and Mandibular Advancement Devices in Obstructive Sleep Apnea. Int J Head Neck Surg 2019;10(2):31-33.

Source of support: Nil

Conflict of interest: None

- As a temporary alternative to CPAP when traveling or when there is no electricity (e.g., camping sites),

- In combination with CPAP to reduce pressures

- As a predictor of success of bimaxillary advancement surgery. OAs can be classified as per their mechanism of action, customization, and adjustability as follows:

- Mechanism of action

- Tongue-retaining

- Mandibular-repositioning

- Combination of mandibular-repositioning with CPAP

- Customization

- Customized

- Non-customized

- Adjustability

- Adjustable/titratable

- Non-adjustable/non-titratable

Tongue-retaining appliances: These appliances hold the tongue forward in the bulb of the appliance, resulting in the moderate advancement of the mandible. They are indicated where there is a lack of tooth support or edentulous, non-apneic snorers, mild snoring, Down syndrome. Adverse effects of tongue-retaining appliances include excessive salivation and difficulty in swallowing. Examples of tongue-retaining appliances in the market include Aveo TSD, SnorX, TRD, Deepsleep Pro, etc. (Fig. 1).

Mandibular-repositioning appliances: These appliances hold the mandible in a therapeutic position to stabilize and dilate the upper airway, particularly at the level of the velopharynx, thereby reducing upper airway collapse. They are indicated where the teeth support is adequate for attaining maximum retention of the appliance. The device results in enlargement of the retropalatal or velopharyngeal airway while the mandible is protruded. ${ }^{1}$ Adverse effects of mandibular-repositioning appliances include tooth soreness, gum soreness, occlusal changes, and discomfort of the temporomandibular joint. Examples of mandibular-repositioning appliances in the market include Herbst, Thornton adjustable

() The Author(s). 2019 Open Access This article is distributed under the terms of the Creative Commons Attribution 4.0 International License (https://creativecommons. org/licenses/by-nc/4.0/), which permits unrestricted use, distribution, and non-commercial reproduction in any medium, provided you give appropriate credit to the original author(s) and the source, provide a link to the Creative Commons license, and indicate if changes were made. The Creative Commons Public Domain Dedication waiver (http://creativecommons.org/publicdomain/zero/1.0/) applies to the data made available in this article, unless otherwise stated. 
Dental Appliances and Mandibular Advancement Devices in Obstructive Sleep Apnea

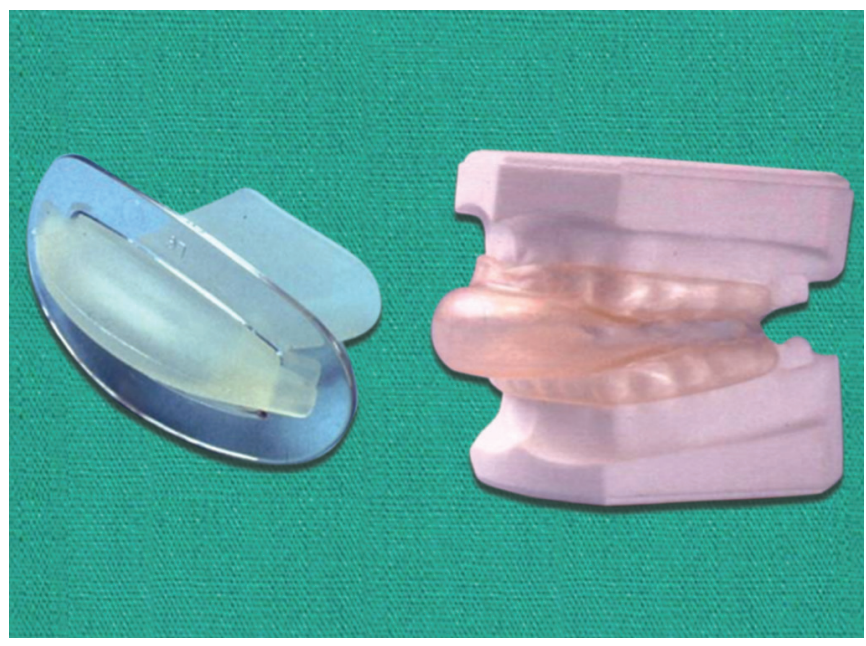

Fig. 1: Tongue-retaining appliances
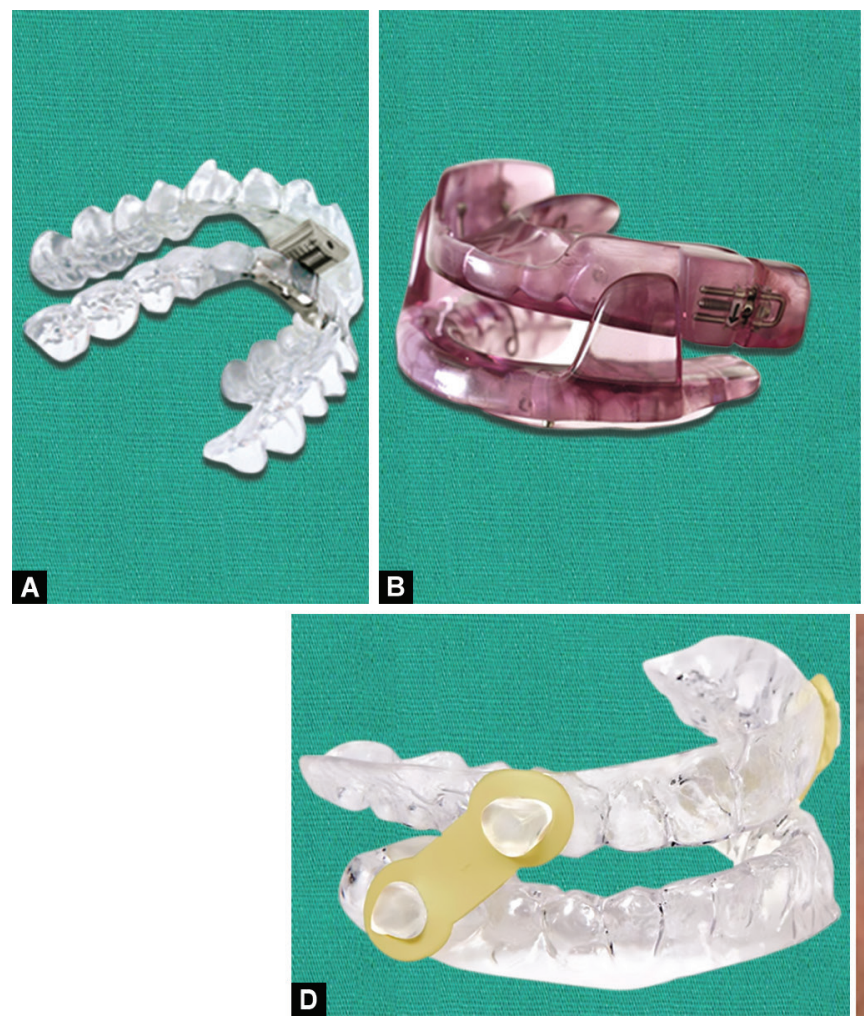

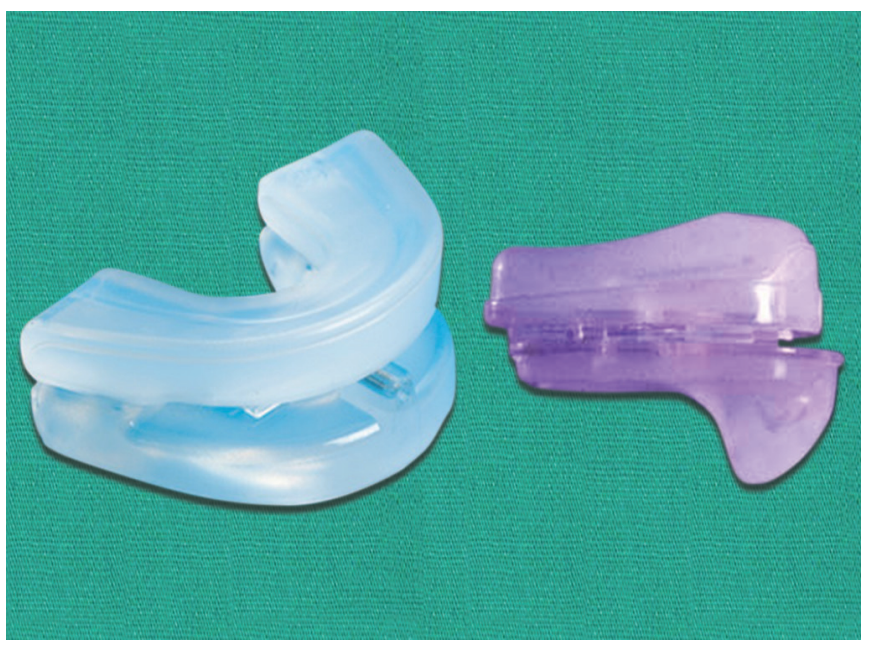

Fig. 2: Mandibular repositioning appliances (Non-adjustable)
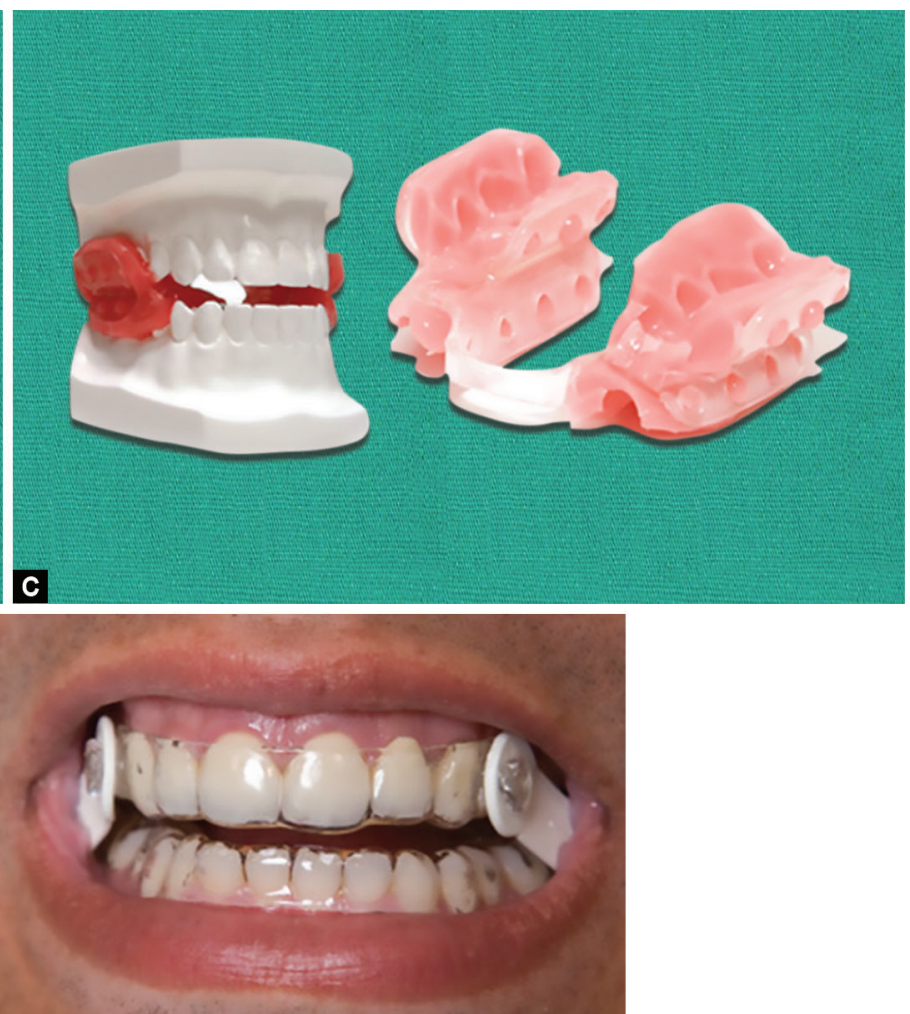

Figs 3A to D: Mandibular repositioning appliances (Adjustable). (A) Thornton adjustable positioner (TAP); (B) Somnomed; (C) Silent sleep; (D) EMA

Positioner, Elastic Mandibular Advancement, Silent Sleep, Somnomed, etc. (Figs 2 and 3).

Combination of mandibular-repositioning with CPAP: A recent advancement is a combination of a repositioner along with CPAP, e.g., the Sleep Apnea Airway Management System (SAAMS ${ }^{\mathrm{T}}$ ), which uses a combination of the TAP ${ }^{\circledR}$ oral appliance and standard CPAP technology. Such devices reduce CPAP pressure and eliminate the requirement of straps, which increase patient compliance.

\section{Custom-made vs Non-customized Appliances}

Custom appliances are more retentive as they are fabricated according to the teeth size; the retention of non-custom appliances is uncertain. Custom appliances can easily be titrated for patient efficacy, whereas the non-custom ones cannot. Custom ones last longer than the non-custom ones. ${ }^{1}$ Non-custom ones cannot be used where there is crowding of teeth. Flowchart 1 shows a treatment protocol or algorithm, which should be followed to decide when and how dental appliances should be used by sleep physicians.

\section{Treatment Protocol}

Side effects and complications of OA therapy. OA therapy can have adverse effects affecting the facial structures, teeth, bite position, and the tongue. Almost all the complications are temporary 
Flowchart 1: Treatment protocol for dental appliances

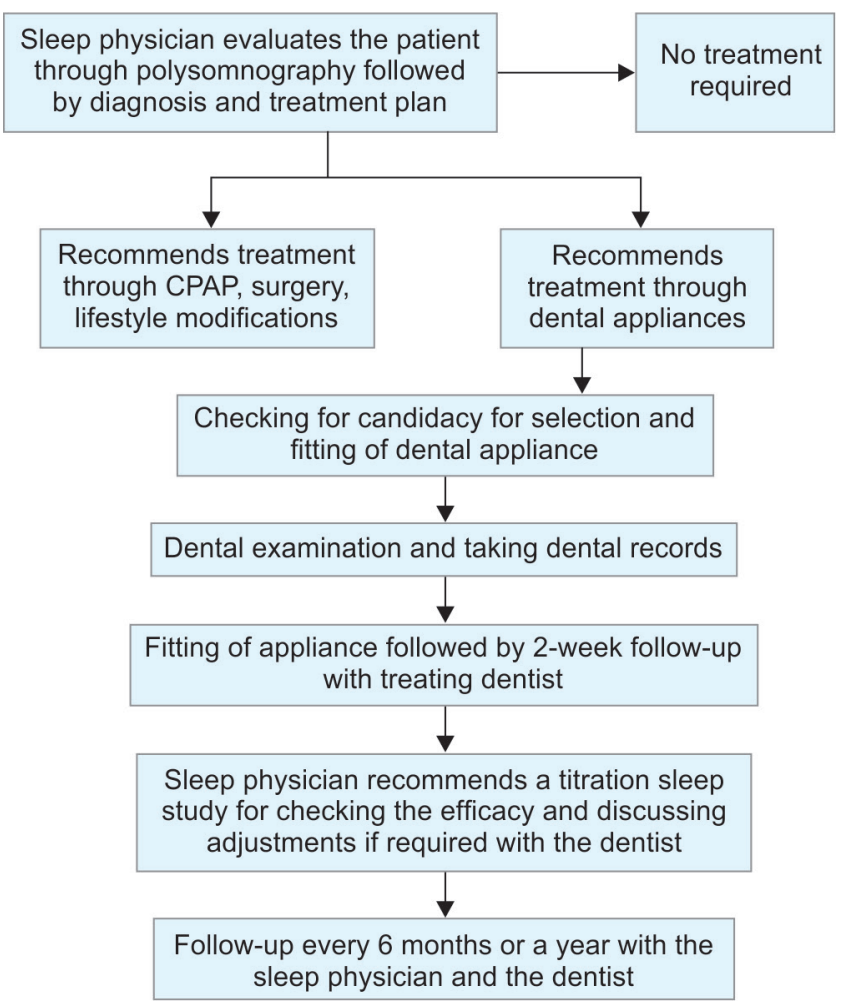

(resolving within 2 months) and require close communication with the treating dentist to avoid permanent complications. ${ }^{1}$

- Affecting facial structures: TMJ pain, soreness in cheek muscles, excessive salivation, dryness of mouth

- Affecting teeth: Gum soreness, tooth soreness, tooth mobility

- Affecting occlusion/normal relaxed bite position: Inability to close the mouth, spaces between teeth (diastema), decrease in overjet and overbite, decreased occlusion contact, etc.

- Affecting tongue: Soreness

The AASM and the American Academy of Dental Sleep Medicine (AADSM) in 2015 released updated guidelines for the use of OAs in the treatment of OSA and snoring. ${ }^{4}$

- For adult patients with snoring (and no OSA), sleep physicians should prescribe OAs rather than no treatment.
- When OA therapy is prescribed by a sleep physician for OSA, a qualified dentist should be consulted to prepare a custom, titratable appliance; this should be preferred to noncustomized devices.

- For adult patients with OSA who cannot tolerate CPAP therapy or wish for an alternative, sleep physicians should prescribe OAs.

- After patients with OSA begin using OAs, dentists should schedule regular follow-ups to check for dental side effects or occlusal changes and reduce their incidence.

- In addition, sleep physicians too should conduct follow-up sleep testing for treatment efficacy in these patients.

- Sleep physicians who are treating patients with OSA should be aware of and recommend consultations with qualified dentists, and vice versa.

\section{Pearls of Wisdom}

OAs are a good alternative to CPAP in patients with mild to moderate OSA.

- Patient compliance is better with OAs than with CPAP.

- OAs can be tongue-retaining type or mandibular-repositioning type and can be custom-made or non-customised.

- Custom-made, titratable devices are smaller, more duarable, and may have better adherence.

- Sleep physician and qualified dentist should both be ideally involved in the treatment and follow-up of OSA patients with OAs.

\section{References}

1. Jacobowitz O. Advances in Oral Appliances for Obstructive Sleep Apnea. InSleep-Related Breathing Disorders 2017. 80:57-65. Karger Publishers.

2. Wolfgang Schmidt-Nowara, Lowe A, et al. Oral Appliances for the Treatment of Snoring and Obstructive Sleep Apnea: A Review; Sleep, 1995; 18(6):501-510

3. Robin P. Glossoptosis due to atresia and hypotrophy of the mandible. Am J Dis Child. 1934;48:541-547.

4. Ramar K, Dort LC, et al. Clinical practice guideline for the treatment of obstructive sleep apnea and snoring with oral appliance therapy: an update for 2015. Journal of clinical sleep medicine. $2015 \mathrm{Jul}$ 15;11(07):773-827.

5. Practice Parameters for the Treatment of Snoring and Obstructive Sleep Apnea with Oral Appliances: An Update for 2005 An American Academy of Sleep Medicine Report. 